International Journal of Electrical Engineering and Technology (IJEET)

Volume 12, Issue 9, September 2021, pp. 43-54, Article ID: IJEET_12_09_005

Available online at https://iaeme.com/Home/issue/IJEET? Volume $=12 \&$ Issue $=9$

ISSN Print: 0976-6545 and ISSN Online: 0976-6553

DOI: https://doi.org/10.34218/IJEET.12.9.2021.005

\title{
COMPARATIVE STUDY ON THE COST ANALYSIS OF SOFTWARE DEVELOPMENT MODEL APPLICABLE TO SYSTEM SOLUTIONS BASED ON GAMMA FAMILY DISTRIBUTION
}

\author{
Jong-Man Kim ${ }^{1 *}$ and Yu-Jeong Yang ${ }^{2}$ \\ ${ }^{1}$ Professor, International Cooperation Center, \\ Namseoul University, Cheonan, Korea \\ ${ }^{2}$ Undergraduate Student, Department of Elderly Welfare \& Department of Smart Care, \\ Namseoul University, Cheonan, Korea \\ *Corresponding Author
}

\begin{abstract}
In this study, we analyzed and predicted the software development cost applicable to system solutions based on the lifetime distribution of the Gamma family (Erlang and Log-Logistic) which is widely utilized in the field of software reliability testing together with the Goel-Okumoto basic model. Also, the optimal development cost model was suggested through these analysis results. For this, software failure time data was used, the parametric calculation was applied to the maximum likelihood estimation (MLE) method, and nonlinear equations were solved by a numerical method (bisection method). As a result, First, the cost curve shape of all proposed models decreased in the initial stage but continuously increased in the later stage because the probability of finding the remaining defects is gradually lowered. Second, if the cost of eliminating a single defect found in the testing process increased, the development cost also increased, but the release time did not change. Third, if the defect correction cost discovered by the operator during the operation stage increased, the development cost at the time of release is increased, and the release time is also delayed. Also, it was confirmed that the Erlang distribution model showed the best performance because it has a lower software development cost and a faster release time point than the proposed other models. Through this study, the software development cost of the Gamma family distribution without the existing research examples was newly analyzed. Therefore, we were able to present software developers with the necessary information to predict the economic software development cost and the optimal release time.
\end{abstract}

Key words: Cost Analysis, Erlang, Gamma Family, Log-Logistic, Software Development Model, System Solutions 
Comparative Study on the Cost Analysis of Software Development Model Applicable to System Solutions Based on Gamma Family Distribution

Cite this Article: Jong-Man Kim and Yu-Jeong Yang. Comparative Study on the Cost Analysis of Software Development Model Applicable to System Solutions Based on Gamma Family Distribution. International Journal of Electrical Engineering and Technology (IJEET). 12(9). 2021, pp. 43-54. https://iaeme.com/Home/issue/IJEET?Volume $=12 \&$ Issue $=9$

\section{INTRODUCTION}

Along with the rapid development of software convergence technology, the need for developing reliable high-quality software that can process various big data accurately without failure is also increasing. In particular, the most important task in this software development process applicable to system solutions is to find the most economical cost through analysis and prediction of development cost based on the software reliability. For this reason, software reliability models using the non-homogeneous Poisson process (NHPP) have been extensively studied to improve software quality. In particular, many NHPP software reliability models using the intensity function and the mean value function have been proposed to estimate the performance attributes such as the failure occurring rate and the future reliability in a controlled test environment [1]. Concerning the NHPP reliability model, Goel and Okumoto [2] proposed an exponential type software reliability model, Huang [3] explained the software reliability attributes using the mean value function, Pham [4] proposed a distribution function and its application in software reliability. Also, Kim [5] analyzed the cost of the software development model based on the Burr-Hatke exponential distribution model. Also, Pham and Zhang [6] proposed a new reliability model based on NHPP software reliability and cost with testing coverage, and Kim [7] proposed a comparative study on the performance analysis of software development cost model based on inverse Exponential distribution. Also, Yang [8] analyzed the cost attributes of the software development model based on Lindley-type distribution.

Therefore, in this study, after applying the Gamma family distribution (Erlang and LogLogistic) model widely used in the software reliability field together with Goel-Okumoto basic model to the software development cost model, we newly analyze the attributes of cost and release time of the proposed models and will present the best cost model among the Gamma family distribution applicable to system solutions.

\section{RELATED RESEARCH}

\subsection{NHPP Software Reliability Model}

The NHPP reliability model is a model that measures the reliability with the average failure rate function by the number of failures generated per unit time.

$\mathrm{N}(\mathrm{t})$ is the cumulative failures of the software that can be detected up to time $t, m(t)$ is a mean value function when $\lambda(\mathrm{t})$ is expressed by an intensity function, and thus the cumulative failure number $\mathrm{N}(\mathrm{t})$ follows a Poisson probability density function having a parameter $\mathrm{m}(\mathrm{t})$.

That is

$$
\mathrm{P}\{N(t)=n\}=\frac{[m(t)]^{n} \cdot e^{-m(t)}}{n !}
$$

Note that $n=0,1,2, \cdots \infty$.

The mean value function and the intensity function of the NHPP model are as follows.

$$
\mathrm{m}(\mathrm{t})=\int_{0}^{t} \lambda(s) d s
$$




$$
\frac{d m(t)}{d(t)}=\lambda(t)
$$

In terms of software reliability, the mean value function represents a software failure occurrence expected value, the intensity function is the failure rate function, and means the failure occurring rate per defect. Also, the time domain NHPP models are classified into a finite failure that the failure does not occur during repairing the failure, and an infinite failure that the failure can occur at the time of repairing failure.

In this study, we will analyze the software reliability based on finite failure cases. If the expected value of the failure that can be found up to time $[0, t]$ is $\theta$, then the mean value function and the intensity function are as follows.

$$
\begin{aligned}
& \mathrm{m}(\mathrm{t} \mid \theta, \mathrm{b})=\theta F(\mathrm{t}) \\
& \lambda(\mathrm{t} \mid \theta, \mathrm{b})=\theta F(\mathrm{t})^{\prime}=\theta f(t)
\end{aligned}
$$

If using Eq. 4 and Eq. 5, the likelihood function of the finite-failure NHPP model is derived as follows.

$$
L_{N H P P}(\Theta \mid \underline{x})=\left(\prod_{i=1}^{n} \lambda\left(x_{i}\right)\right) \exp \left[-m\left(x_{n}\right)\right]
$$

Note that $\underline{x}=\left(x_{1}, x_{2}, x_{3} \cdots x_{n}\right)$.

\subsection{Goel-Okumoto Basic: NHPP Model}

The Goel-Okumoto model is a well-known Gamma basic model in the software reliability field. Let $f(t)$ and $F(t)$ for the Goel-Okumoto model be a probability density function and a cumulative distribution function, respectively. Assuming that the failure occurring expected value of the observation point $[0, t]$ is $\theta$, then the mean value function and the intensity function are as follows [9].

$$
\begin{aligned}
& \mathrm{m}(\mathrm{t} \mid \theta, \mathrm{b})=\theta F(\mathrm{t})=\theta\left(1-e^{-b t}\right) \\
& \lambda(\mathrm{t} \mid \theta, \mathrm{b})=\theta \mathrm{f}(\mathrm{t})=\theta \mathrm{b} e^{-b t}
\end{aligned}
$$

Note that $\theta>0, b>0$.

If using Eq. 7 and Eq. 8, the likelihood function of the finite-failure NHPP model is derived as follows.

$$
L_{N H P P}(\theta, \mathrm{b} \mid \underline{x})=\left(\prod_{i=1}^{n} \theta b e^{-b x_{i}}\right) \exp \left[-\theta\left(1-e^{-b x_{n}}\right)\right]
$$

Note that $\underline{x}=\left(0 \leq x_{1} \leq x_{2} \leq \cdots \leq x_{n}\right)$.

The log-likelihood function, if using Eq. 9, is simplified to the following log conditional equation.

$$
\ln L_{N H P P}(\Theta \mid \underline{x})=n \ln \theta+n \ln b-b \sum_{k=1}^{n} x_{k}-\theta\left(1-e^{-b x_{n}}\right)
$$

Therefore, the maximum likelihood estimator $\hat{\theta}_{M L E}$ and $\hat{b}_{M L E}$ satisfying the following Eq. 11 and Eq. 12 can be calculated by the bisection method. 
Comparative Study on the Cost Analysis of Software Development Model Applicable to System Solutions Based on Gamma Family Distribution

$$
\begin{aligned}
& \frac{\partial \ln L_{N H P P}(\Theta \mid \underline{x})}{\partial \theta}=\frac{n}{\hat{\theta}}-1+e^{-\hat{b} x_{n}}=0 \\
& \frac{\partial \ln L_{N H P P}(\Theta \mid \underline{x})}{\partial b}=\frac{n}{\hat{b}}-\sum_{i=1}^{n} x_{n}-\hat{\theta} x_{n} e^{-\hat{b} x_{n}}=0
\end{aligned}
$$

\subsection{Erlang Distribution: NHPP Model}

The Erlang distribution is the lifetime distribution widely used in the reliability field and belongs to the Gamma family. The probability density function and the cumulative distribution function considering the shape parameter (a) and the scale parameter (b) are as follows [10].

$$
\begin{aligned}
& \mathrm{f}(\mathrm{t})=\frac{b^{a}}{\Gamma(a)} t^{a-1} e^{-b t} \\
& F(\mathrm{t})=\left(1-e^{-b t} \sum_{i=0}^{a-1} \frac{(b t)^{i}}{i !}\right)
\end{aligned}
$$

Note. $a, b>0, a=1,2,3 \cdots, t \in[0, \infty]$

Therefore, the mean value function and the intensity function of the finite fault NHPP Erlang model are as follows.

Note that $\theta>0, \mathrm{~b}>0$.

$$
\begin{aligned}
& \mathrm{m}(\mathrm{t} \mid \theta, \mathrm{b})=\theta F(\mathrm{t})=\theta\left(1-e^{-b t} \sum_{i=0}^{a-1} \frac{(b t)^{i}}{i !}\right) \\
& \lambda(\mathrm{t} \mid \theta, \mathrm{b})=\theta \mathrm{f}(\mathrm{t})=\frac{b^{a}}{\Gamma(a)} t^{a-1} e^{-b t^{i=0}}
\end{aligned}
$$

In this study, we will analyze the case where the shape parameter (a) is 2 . Therefore, it can be derived as follows.

$$
\begin{gathered}
\ln L_{N H P P}(\Theta \mid \underline{x})=n \ln \theta-n \ln \Gamma(a)+n a \ln b+(a-1) \sum_{i=1}^{n} \ln x_{i}-b \sum_{i=1}^{n} x_{i} \\
-\theta+\theta e^{-b x_{n}}\left(\sum_{i=0}^{a-1} \frac{\left(b x_{n}\right)^{i}}{i !}\right)
\end{gathered}
$$

Note. $\underline{x}=\left(0 \leq x_{1} \leq x_{2} \leq \cdots \leq x_{n}, \Theta\right.$ is parameter space.

Therefore, the maximum likelihood estimator $\hat{\theta}_{M L E}$ and $\hat{b}_{M L E}$ can be calculated by the bisection method as follows.

$$
\begin{aligned}
& \frac{\partial \ln L_{N H P P}(\Theta \mid \underline{x})}{\partial \theta}=\frac{n}{\theta}-1+e^{-b x_{n}}\left(1+b x_{n}\right)=0 \\
& \frac{\partial \ln L_{N H P P}(\Theta \mid \underline{x})}{\partial b}=\frac{2 n}{b}-\sum_{i=1}^{n} x_{i}-\theta b x_{n}{ }^{2} e^{-b x_{n}}=0
\end{aligned}
$$

Note. $x=\left(x_{1}, x_{2}, x_{3} \cdots x_{n}\right)$. 


\subsection{Log-Logistic Distribution: NHPP Model}

The Log-Logistic distribution has a property that increases and decreases in the form of failure occurring rate and thus is widely applied in the reliability field, and belongs to the Gamma family. The probability density function and the cumulative distribution function considering the shape parameter $(\mathrm{k})$ are as follows [11].

$$
\begin{aligned}
& \mathrm{f}(\mathrm{t} \mid \tau, \mathrm{k})=\frac{\tau k(\tau t)^{k-1}}{\left[1+(\tau t)^{k}\right]^{2}} \\
& \mathrm{~F}(\mathrm{t} \mid \tau, \mathrm{k})=\frac{(\tau t)^{k}}{\left[1+(\tau t)^{k}\right]}
\end{aligned}
$$

Note that $\tau>0, k>0$

Therefore, the mean value function and the intensity function of the finite fault NHPP LogLogistic model are as follows.

$$
\begin{aligned}
& \mathrm{m}(\mathrm{t} \mid \theta, \tau, \mathrm{k})=\theta F(\mathrm{t})=\theta \frac{(\tau t)^{k}}{\left[1+(\tau t)^{k}\right]} \\
& \lambda(\mathrm{t} \mid \theta, \tau, \mathrm{k})=\theta \mathrm{f}(\mathrm{t})=\theta \frac{\tau k(\tau t)^{k-1}}{\left[1+(\tau t)^{k}\right]^{2}}
\end{aligned}
$$

Note that $\theta>0, \tau, \mathrm{k}>0$.

Because the Log-Logistic distribution has a form in which the failure rate increases, the case where the shape parameter $(\mathrm{k})$ that determines the form of the failure lifetime distribution is 2 was applied. Therefore, it can be derived as follows.

$\ln L_{N H P P}(\Theta \mid \underline{x})=n \ln 2+n \ln \theta+2 n \ln \tau$

$$
+\sum_{i=1}^{n} x_{i}-2 \sum_{i=1}^{n} \ln \left[1+\left(\tau x_{i}\right)^{2}\right]-\theta \frac{\left(\tau x_{n}\right)^{2}}{\left[1+\left(\tau x_{n}\right)^{2}\right]}=0
$$

Therefore, the maximum likelihood estimator $\hat{\theta}_{M L E}$ and $\hat{\tau}_{M L E}$ can be calculated by the bisection method as follows.

$$
\begin{array}{r}
\frac{\partial \ln L_{N H P P}(\Theta \mid \underline{x})}{\partial \theta}=\frac{n}{\hat{\theta}}-\frac{\left(\hat{\tau} x_{n}\right)^{2}}{\left[1+\left(\hat{\tau} x_{n}\right)^{2}\right]}=0 \\
\frac{\partial \ln L_{N H P P}(\Theta \mid \underline{x})}{\partial \tau}=\frac{2 n}{\hat{\tau}}-2 \hat{\tau} \sum_{i=1}^{n} x_{i}{ }^{2} \frac{1}{\ln \left[1+\left(\hat{\tau} x_{i}\right)^{2}\right]} \\
-\hat{\theta}\left(\frac{2 \hat{\tau} x_{n}{ }^{2}\left(1+\hat{\tau}^{2} x_{n}-\hat{\tau}^{2} x_{n}{ }^{2}\right.}{\left[1+\left(\hat{\tau} x_{n}\right)^{2}\right]^{2}}\right)=0
\end{array}
$$

Note that $x=\left(x_{1}, x_{2}, x_{3} \cdots x_{n}\right)$.

\subsection{Software Development Model Applicable to System Solution}

The software development model applicable to system solutions is as follows [12].

$$
\begin{aligned}
& \mathrm{E}_{\mathrm{t}}=E_{1}+E_{2}+E_{3}+E_{4} \\
& =E_{1}+C_{2} \times t+C_{3} \times m(t)+C_{4} \times\left[m\left(t+t^{\prime}\right)-m(t)\right]
\end{aligned}
$$


Note that $\mathrm{E}_{\mathrm{t}}$ is the estimated total cost of software development, $m(t)$ is the mean value function of the proposed Gamma family distribution, and $t^{\prime}$ is the time for operating and maintaining the software after releasing the developed software.

\subsubsection{Mean Value Function ( $m(t))$}

The method of calculating the mean value function of the proposed software development model (Gamma family distribution) that determines the software development cost is shown in Table 2. Also, $m(t)$ is the mean value function, and means the expected value of the failure that can be detected at time $t$.

Table 1 Mean value function of the proposed software development cost model

\begin{tabular}{|l|c|c|}
\hline $\begin{array}{c}\text { NHPP model } \\
\text { (Shape Parameter) }\end{array}$ & $\begin{array}{c}\boldsymbol{m}(\boldsymbol{t}) \text { of Gamma Family } \\
\text { Distribution }\end{array}$ & $\begin{array}{c}\text { m(t) of Software } \\
\text { Development Model }\end{array}$ \\
\hline Goel-Okumoto & $m(t)=\hat{\theta}\left(1-e^{-\hat{b} t}\right)$ & \multirow{2}{*}{$E_{3}=C_{3} \times m(t)$} \\
\hline $\begin{array}{l}\text { Erlang } \\
(\mathrm{a}=2)\end{array}$ & $m(t)=\hat{\theta}\left(1-e^{-\hat{b} t}[1+(\hat{b} t)]\right)$ & $E_{4}=C_{4} \times\left[m\left(t+t^{\prime}\right)-m(t)\right]$ \\
\cline { 1 - 2 } $\begin{array}{l}\text { Log-Logistic } \\
(\mathrm{k}=2)\end{array}$ & $m(t)=\hat{\theta} \frac{(\hat{\tau} t)^{2}}{\left[1+(\hat{\tau} t)^{2}\right]}$ & \\
\hline
\end{tabular}

\subsubsection{Development Cost Attributes $\left(E_{1}, E_{2}, E_{3}, E_{4}\right)$}

Each cost attributes that constituting the total software development cost is as follows.

(1) $E_{1}$ represents the software design and initial software development costs and is considered a constant.

(2) $E_{2}$ represents the software testing cost per unit time and can be derived as follows.

$$
E_{2}=C_{2} \times t
$$

Note that $C_{2}$ is the testing cost, $\mathrm{t}$ is the testing time point.

(3) $E_{3}$ represents the cost of removing a defect by detecting a basic defect and can be derived as follows.

$$
E_{3}=C_{3} \times m(t)
$$

Note that $C_{3}$ is the cost of removing one defect found in the testing process.

(4) $E_{4}$ represents the cost of eliminating all remaining defects in the operating software system and can be derived as follows.

$$
E_{4}=C_{4} \times\left[m\left(t+t^{\prime}\right)-m(t)\right]
$$

Note that $C_{4}$ is the defect correction cost detected by the operator at the software operation stage after the software is released.

In reality, $C_{4}$ has a higher cost than $C_{2}$ and $C_{3}$. Therefore, this study is applied to the real situation that $C_{4}$ is higher than $C_{2}$ and $C_{3}$.

Therefore, the optimal software release time for the software development cost can be derived as follows. 


$$
\frac{\partial \mathrm{E}_{\mathrm{t}}}{\partial t}=\mathrm{E}^{\prime}=\left(\mathrm{E}_{1}+E_{2}+E_{3}+E_{4}\right)^{\prime}=0
$$

That is, the optimal release time is the time point at which the lowest development cost is satisfied.

\section{SYSTEM SOLUTIONS USING SOFTWARE FAILURE TIME DATA}

In this paper, we will analyze the software development cost of the proposed models using software failure time data [13] as shown in Table 2. Therefore, these analysis results can be applied for big data utilization in various related fields. This software failure time is the data that was occurred 30 times in 738.68 unit time.

Table 2 Software Failure Time Data

\begin{tabular}{|c|c|c|c|c|c|}
\hline Failure & Failure & Failure Time & Failure & Failure Time & Failure Time \\
\hline 1 & 30.02 & 30.02 & 16 & 151.78 & 15.53 \\
\hline 2 & 31.46 & 1.44 & 17 & 177.50 & 25.72 \\
\hline 3 & 53.93 & 22.47 & 18 & 180.29 & 2.79 \\
\hline 4 & 55.29 & 1.36 & 19 & 182.21 & 1.92 \\
\hline 5 & 58.72 & 3.43 & 20 & 186.34 & 4.13 \\
\hline 6 & 71.92 & 13.20 & 21 & 256.81 & 70.47 \\
\hline 7 & 77.07 & 5.15 & 22 & 273.88 & 17.07 \\
\hline 8 & 80.90 & 3.83 & 23 & 277.87 & 3.99 \\
\hline 9 & 101.90 & 21.00 & 24 & 453.93 & 176.06 \\
\hline 10 & 114.87 & 12.97 & 25 & 535.00 & 81.07 \\
\hline 11 & 115.34 & 0.47 & 26 & 537.27 & 2.27 \\
\hline 12 & 121.57 & 6.23 & 27 & 552.90 & 15.63 \\
\hline 13 & 124.97 & 3.40 & 28 & 673.68 & 120.78 \\
\hline 14 & 134.07 & 9.10 & 29 & 704.49 & 30.81 \\
\hline 15 & 136.25 & 2.18 & 30 & 738.68 & 34.19 \\
\hline
\end{tabular}

Laplace trend test was utilized to verify the software failure time data as shown in Figure 1.

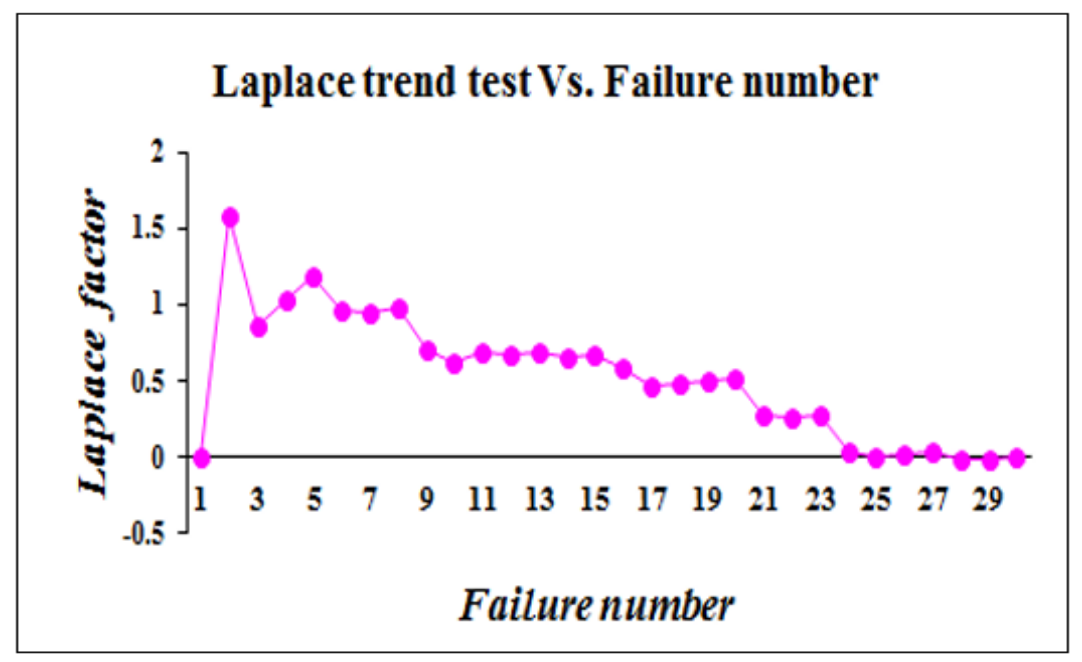

Figure 1 Estimation results of Laplace Trend Test

In general, if the Laplace factor estimates are distributed between -2 and 2, the data are reliable because the extreme values do not exist and are stable [14]. 
As a result of this test, the estimated value of the Laplace factor was distributed between 0 and 2. That is, it is possible to apply this data because there is no extreme value.

In this study, the maximum likelihood estimation (MLE) method was applied to calculate parameter estimation [15]. Its results are shown in Table 2.

Table 3. Parameter estimation results of the proposed model

\begin{tabular}{|l|l|c|c|}
\hline \multicolumn{1}{|c|}{ Type } & \multicolumn{1}{c|}{$\begin{array}{c}\text { NHPP Model } \\
\text { (Shape Parameter) }\end{array}$} & \multicolumn{2}{c|}{ MLE } \\
\hline Gamma & Goel-Okumoto & $\hat{\theta}=33.4092$ & $\hat{b}=0.3090$ \\
\hline \multirow{3}{*}{ Gamma family } & Erlang & $\hat{\theta}=30.5978$ & $\hat{b}=0.7922$ \\
\cline { 2 - 4 } & Log-Logistic & $\hat{\theta}=32.2412$ & $\hat{\tau}=0.4953$ \\
\hline
\end{tabular}

Explanatory notes. MLE = Maximum Likelihood Estimation

In this study, we assumed the cost of software development as [Supposition 1] [Supposition 4] to simulate the same as the actual software development conditions. To do this, we will analyze software development costs by changing each cost component $\left(C_{2}, C_{3}, C_{4}\right)$ that constituting the total software development cost $\left(E_{t}\right)$.

\section{[Supposition 1: Basic conditions]}

$$
E_{1}=40 \$, C_{2}=5 \$, C_{3}=1.5 \$, C_{4}=10 \$, t^{\prime}=40 \text { (hours) }
$$

The result of the cost curve using [Supposition 1] is as shown in Figure 2. The development cost curve showed a constant pattern for a short period after showing a decreasing pattern in the initial stage, but it showed an increasing pattern as the release time passes.

During the initial stage, the development cost is decreased because the number of defects inherent in the software is reduced. But the development cost is increased because the probability of finding the remaining defects during the later stage is gradually lowered. As a result, the pattern of the development cost curve gradually increases as the release time passes [16].

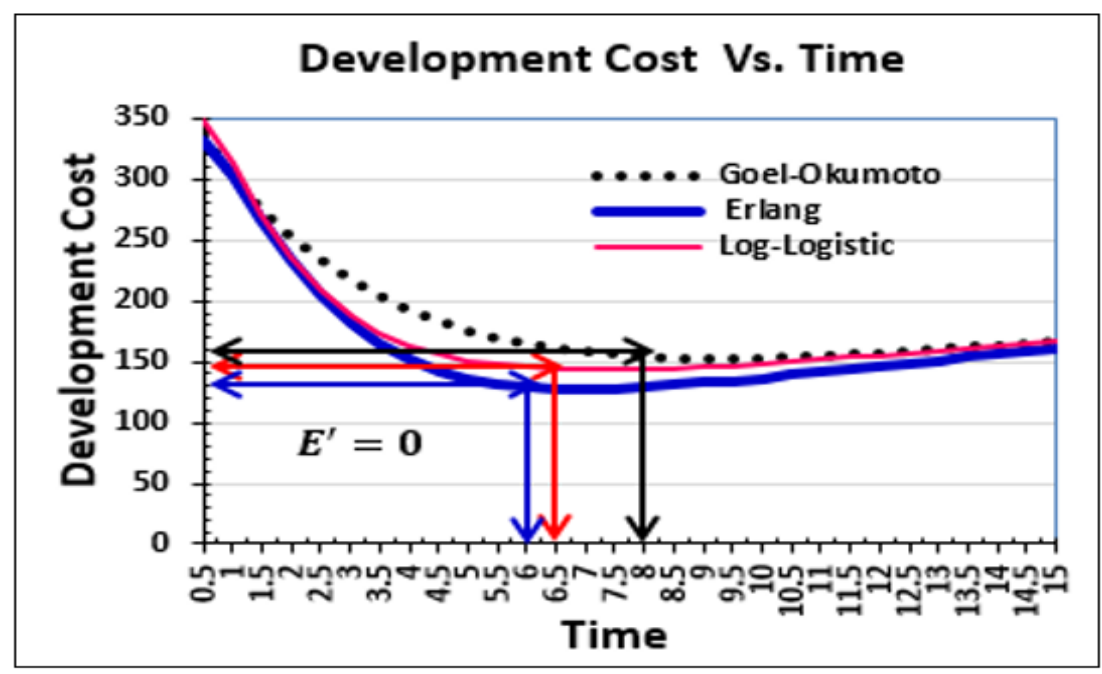

Figure 2. The software development cost curve simulated to the condition of [Supposition 1]

As shown in Figure 2, the proposed models showed a similar pattern, but the Erlang model was more efficient because it has a lower development cost and faster release time than other models (Go-Okumoto and Log-Logistic). 
[Supposition 2: Assumed that only cost $C_{2}$ is increased in Supposition 1]

$$
E_{1}=40 \$, C_{2}=10 \$, C_{3}=1.5 \$, C_{4}=10 \$, t^{\prime}=40 \text { (hours) }
$$

[Supposition 2] is a case where the software testing cost $\left(C_{2}\right)$ per unit time is doubled compared with [supposition 1]. As shown in Figure 3, the development cost has increased, but the release time has not changed.

Therefore, it can be seen that fast and precise testing is required so that the testing cost per unit time does not increase before the software is released. Also, the Erlang model is relatively efficient because it has lower development costs and faster release time than other models.

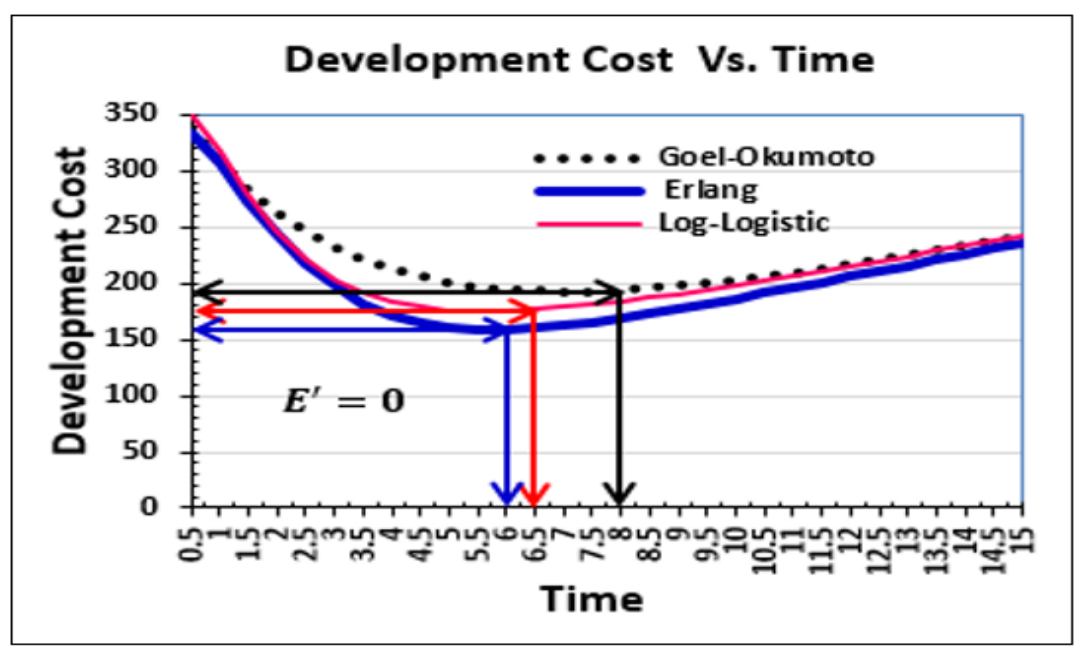

Figure 3. The software development cost curve simulated to the condition of [Supposition 2]

\section{[Supposition 3: Assumed that only cost $C_{3}$ is increased in Supposition 1]}

$$
E_{1}=40 \$, C_{2}=5 \$, C_{3}=3 \$, C_{4}=10 \$, t^{\prime}=40 \text { (hours) }
$$

[Supposition 3] is a case where the $\operatorname{cost}\left(C_{3}\right)$ of removing one defect found in the software testing process is doubled compared to [Supposition 1]. As shown in Figure 4, the development cost has increased, but the release time has not changed. Therefore, in this case, as many defects as possible should be removed at once so that the cost of removing one defect in the software testing step is not increased.

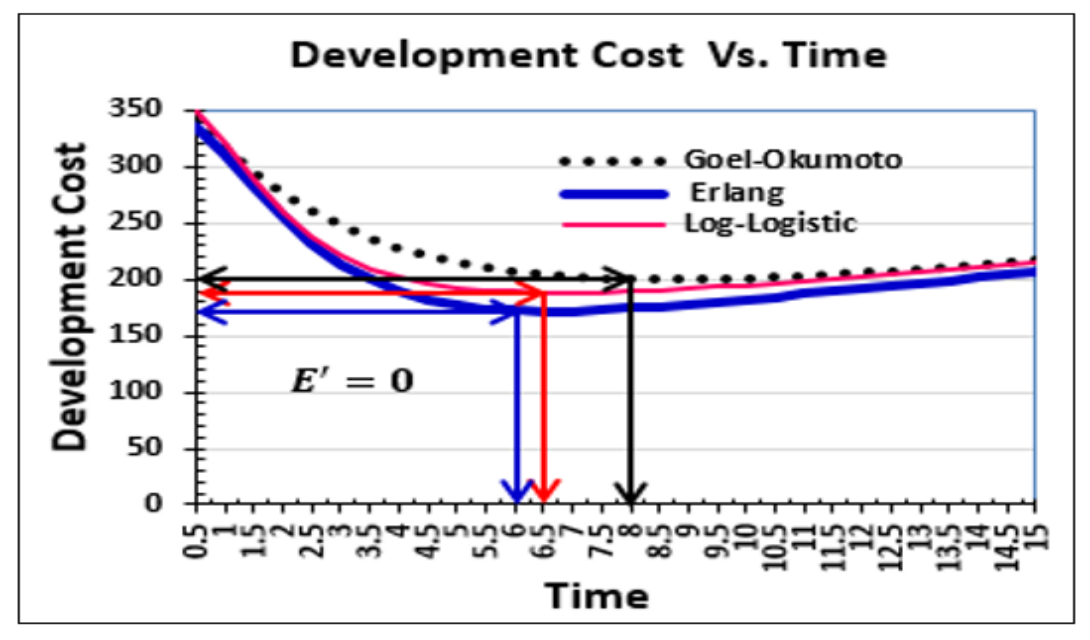

Figure 4. The software development cost curve simulated to the condition of [Supposition 3] 
As shown in the simulation result, the Erlang model is relatively efficient because it has lower development costs and faster release time than other models.

\section{[Supposition 4: Assumed that only cost $C_{4}$ is increased in Supposition 1]}

$$
E_{1}=40 \$, C_{2}=5 \$, C_{3}=1.5 \$, C_{4}=20 \$, t^{\prime}=40 \text { (hours) }
$$

[Supposition 4] is a case where the $\operatorname{cost}\left(C_{4}\right)$ of correcting defects discovered by the software operator during the operation stage after releasing the software is doubled compared to [Supposition 1].

As shown in Figure 5, it showed that the release time is delayed with increasing development costs. Therefore, in this case, we must eliminate all possible defects at the testing stage rather than the operational stage to reduce all defects before releasing the software.

Also, the Erlang model is the best efficient model because it has lower development costs and faster release time than other models. Therefore, it can be seen that the release time is delayed with increasing development costs.

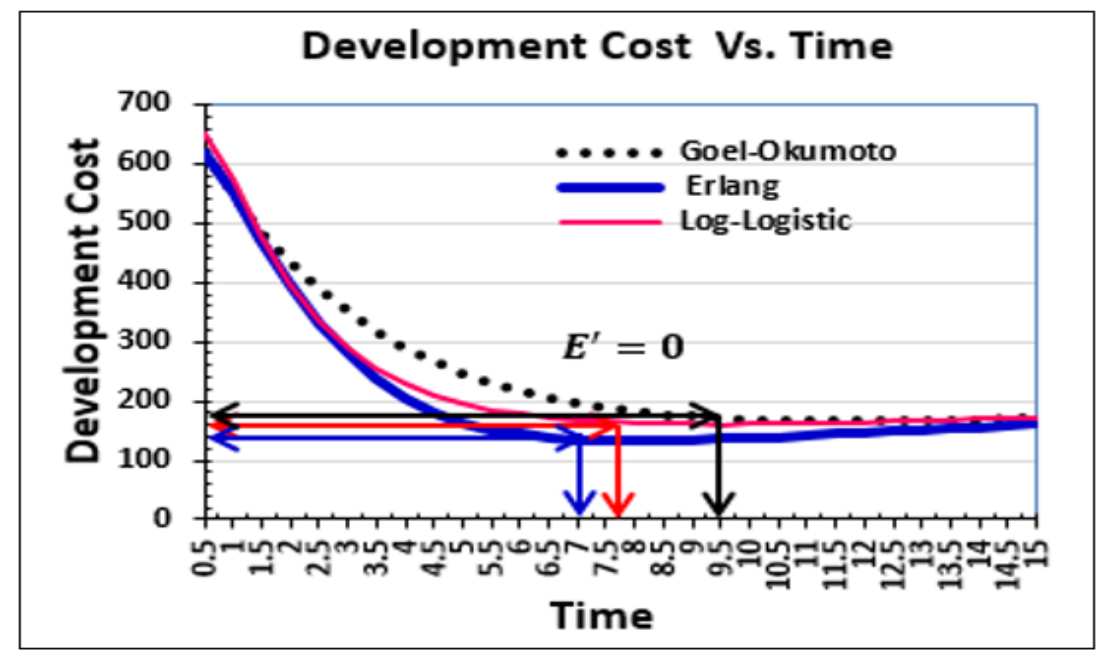

Figure 5. The software development cost curve simulated to the condition of [Supposition 4]

In conclusion, if using this prior information, we can predict the optimal releasing time together with the software development cost, and can also be applied for big data.

\section{CONCLUSIONS}

If software development costs can be quantitatively modeled together with release time during the software development process for system solution, the attributes of development costs can be efficiently analyzed and predicted. Therefore, in this study, based on the Gamma family distribution that can be utilized for system solution analysis, the cost attribute of the software development model was newly analyzed, and the optimal cost model was also presented.

\section{The results of this study can be summarized as follows.}

First, analyzing the basic conditions (Supposition 1), the software development cost curve showed a constant pattern for a short time after a significant decrease in the initial stage but showed a pattern of increasing again in the later stage as the release time passes. The reason is that the number of remaining defects is gradually reduced in the course of removing defects, so the probability of finding residual defects is getting lower. Thus, it can be seen that the cost eventually increases. 
Second, before software release, if the testing cost $\left(C_{2}\right)$ per unit time and the cost $\left(C_{3}\right)$ of removing one defect found in the testing process increases, the development cost has increased as well, but the release time has not changed. But, after software release, if the defect correction $\operatorname{cost}\left(C_{4}\right)$ discovered by the operator increases, the development cost increases, and the release time is also delayed.

Third, the Erlang model showed the best performance because it has low software development cost and fast release time compared to other models (Go-Okumoto and LogLogistic). If using this data, it is possible to provide software developers with the necessary prior information for predicting the optimal release time and the most economical software development costs applicable to system solutions.

Also, further research will be needed to find the optimal software development cost model through analysis with other models having the same type of failure time data.

\section{ACKNOWLEDGEMENTS}

Funding for this paper was provided by Namseoul University.

\section{REFERENCES}

[1] K. Y. Song, I.H. Chang, H. Pham, A Software Reliability Model with a Weibull Fault Detection Rate Function Subject to Operating Environments, Applied Science, 7(983), 2017, 1-16.

[2] Goel A L, Okumoto K, Time-dependent Fault Detection Rate model for Software and Other performance measures, IEEE Transactions on Software Engineering, 28, 1978, 206 -211.

[3] C. Y. Huang, Performance analysis of software reliability growth models with testing effort and change-point, Journal of Systems and Software, 76(2), 2005, 181-194.

[4] H. Pham, Distribution Function and Its Application in Software Reliability, International Journal of Performability Engineering, 15(5), 2019, 1306-1313.

[5] Hee-Cheul Kim, A Comparative Study on the Cost of Software Reliability Model Based on Burr-Hatke Exponential Distribution, International Journal of Engineering Research and Technology, 12(11), 2019, 2036-2040.

[6] H. Pham, X. Zhang, NHPP software reliability and cost models with testing coverage, European Journal of Operational Research, 145, 2003, 443-454.

[7] Hee-Cheul. Kim, A Comparative study on the Cost of Software Development Model Based on Inverse Exponential Distribution, International Journal of Applied Business and Economic Research., 15(14), 2017, 1-7.

[8] Tae-Jin Yang, A Comparative study on the Cost and Release Time of Software Development Model Based on Lindley-Type Distribution, International Journal of Engineering Research and Technology, 13(9), 2020, 2185-2190.

[9] X. Teng, and H. Pham, A new methodology for predicting software reliability in the random field environments, IEEE Transactions on Reliability, 55(3), 2006, 458-468.

[10] Tae-Jin Yang, A Comparative Evaluation on the Performance of Finite Failure NHPP Software Reliability Model Based on Gamma Family Distribution, International Journal of Advanced Research in Engineering and Technology, 12(3), 2021, 371-380. 
Comparative Study on the Cost Analysis of Software Development Model Applicable to System Solutions Based on Gamma Family Distribution

[11] T. R. Adhikari R. S. Srivastava, Poisson-Size biased Lindley Distribution, International Journal of Scientific and Research Publications, 4(1), 2014, 1-6.

[12] Ye Zhang, and Kaigui Wu, Software Cost Model Considering Reliability and Time of Software in Use, Journal of Convergence Information Technology, 7(13), 2012, 135-142.

[13] R. Satya Prasad, K. R. H. Rao, and R. R. L. Kantha, Software Reliability Measuring using Modified Maximum Likelihood Estimation and SPC, International Journal of Computer Applications (0975-8887), 21(7), 2011, 1-5.

[14] Tae-Jin Yang, A Study on the Reliability Performance Analysis of Finite Failure NHPP Software Reliability Model Based on Weibull Life Distribution, International Journal of Engineering Research and Technology. 12(11), 2019, 1890-1896.

[15] Tae-Jin Yang, "Comparative Study on the Performance Attributes of NHPP Software Reliability Model based on Weibull Family Distribution," International Journal of Performability Engineering, 17(4), 2021, 343-353.

[16] P. Sinha, and P. Kumar, An Efficient Approach of Software Cost Estimation Using Soft Computing Technique, International Journal of Computer Engineering \& Technology, 9(4), 2018, 148-158. 